\title{
«La page sortie de mon encrier » : les révélations de Juliette Drouet sur la genèse de l'œuvre de Hugo
}

\section{Florence Naugrette}

\section{(2) OpenEdition}

1 Journals

Édition électronique

URL : http://journals.openedition.org/genesis/2948

DOI : 10.4000/genesis.2948

ISSN : 2268-1590

Éditeur :

Presses universitaires de Paris Sorbonne (PUPS), Société internationale de génétique artistique littéraire et scientifique (SIGALES)

Édition imprimée

Date de publication : 5 décembre 2017

Pagination : 113-123

ISBN : 979-10-231-0580-3

ISSN : 1167-5101

\section{Référence électronique}

Florence Naugrette, « «La page sortie de mon encrier » : les révélations de Juliette Drouet sur la genèse de l'œuvre de Hugo », Genesis [En ligne], 45 | 2017, mis en ligne le 15 décembre 2018, consulté le 11 janvier 2021. URL : http://journals.openedition.org/genesis/2948 ; DOI : https://doi.org/10.4000/ genesis. 2948 


\section{"La page sortie de mon encrier» Les révélations de Juliette Drouet sur la genèse de l'œuvre de Hugo}

Florence Naugrette

Un jour j'écrirai mes mémoires et j'écrirai ce que je sais de vous. Ce sera de curieuses révélations.

7 octobre 1845

L e journal épistolaire de Juliette Drouet, en cours d'édition collective ${ }^{1}$, regorge de renseignements sur la genèse de l'œuvre de Hugo. Tenu de leur rencontre à la mort (1833-1883), il rend compte des (pré)occupations de celle qui fut l'âme sœur, mais aussi la collaboratrice. Compagne, elle vit de son travail d'écrivain, puisqu'il l'entretient; muse de certains poèmes, inspiratrice de personnages de fiction, elle accompagne l'écriture des œuvres, dépouille la presse à leur sortie et assiste aussi souvent que possible aux représentations théâtrales. Collaboratrice, elle réunit de la documentation, veille à certains manuscrits, les copie et prête son concours à la relecture des épreuves. De cette familiarité avec la naissance de l'œuvre, elle rend compte au jour le jour.

Ce témoignage apporte non seulement des confirmations sur ce qu'on sait déjà de la genèse des œuvres de Hugo, mais aussi des informations nouvelles, voire des découvertes. Écrit par la première lectrice, il est puisé sinon à la source de l'écriture, du moins dans ses plus proches parages; déposé dans un journal par lettres, il a le charme de la confidence, de la chose vue et éprouvée, de la trace sédimentée par l'humble récit des travaux et des jours.

\section{Habitudes de travail}

Juliette nourrit avec le travail de son homme une relation ambivalente, faite d'admiration (pour son génie), de reconnaissance (pour le surcroît d'efforts qu'engendre l'entretien d'un «double foyer ${ }^{2} »$ ), de jalousie (ne s'en servirait-il pas comme alibi à son absence ?) et d'inquiétude (pour les risques de surmenage qu'il encourt en se tuant à la tâche).
Selon elle, Hugo travaille comme un titan, particulièrement en exil, où il n'est plus distrait par des mondanités. Le 24 février 1853, elle constate que «depuis [s] a sortie de France [il] n'a pas été un jour sans travailler ${ }^{3} \gg$. Ce labeur est son rival, lorsqu'il empêche Hugo de venir la voir ou de l'emmener en sortie ou en voyage, et son allié quand son amant s'installe chez elle pour écrire ou étudier. Ainsi, pendant la rédaction du Rhin, il poursuit parfois son travail après qu'elle a sombré dans le sommeil, jusqu'à deux ou trois heures du matin 4 . Il lui arrive même de surgir largement après minuit alors qu'elle est déjà endormie, de rester le temps d'écrire avant de repartir en catimini, ce dont elle enrage, le sommeil la privant sinon de sa présence, du moins de son contact. Elle se réjouit inversement des studieux après-midi entiers qu'il passe chez elle, à Paris comme en exil, pendant qu'elle-même copie son œuvre, ou le regarde avec admiration, même si elle se plaint avec humour de sa tendance à accaparer toute la place sur la table 5 .

1. Juliette Drouet, Lettres à Victor Hugo, dir. Florence Naugrette, site du CÉRÉdI, Université de Rouen, ouvert en septembre 2012 : www.juliettedrouet.org. Comité scientifique : Chantal Brière, Jean-Marc Hovasse, Gérard Pouchain, Guy Rosa, Nicole Savy, Françoise Simonet-Tenant. Le corpus intégral sera de 22000 lettres. Je remercie les docteurs qui m'ont signalé les exemples pertinents dans leurs corpus : Bénédicte Duthion, Véronique Heute, Anne Kieffer, Gwenaëlle Sifferlen et Jeanne Stranart.

2. Tel est le titre du livre VIII du premier tome de Jean-Marc Hovasse, Victor Hugo, t. I, Avant l'exil (1802-1851), Paris, Fayard, 2001.

3. Jersey, 24 février 1853 jeudi matin, 8 h. [Bénédicte Duthion/Florence Naugrette (désormais abrégé N.)]. On note entre crochets le nom du transcripteur, éventuellement suivi du nom de son assistant.

4. Elle l'évoquera pourtant avec nostalgie le 30 septembre 1846, époque où il vient plus rarement la voir.

5. Par exemple le 3 novembre 1847 [Sifferlen/N.]. 
Elle ne peut en revanche qu'imaginer comment il travaille chez lui, puisqu'ils vivent séparément jusqu'en 1873. En exil, depuis son logement de La Fallue ${ }^{6}$ d'où elle jouit d'une vue sur Hauteville House, elle émet des suppositions sur les heures de coucher tardives de son compagnon (trahies par ses lumières encore allumées), se demandant si elles sont imputables à l'étude, à l'écriture, à des mondanités ou des galanteries. Quand le signal lui indiquant le réveil de Hugo (un torchon accroché à la balustrade du toit) tarde à apparaitre, elle se demande s'il est en train de travailler dans son lit, comme il en a l'habitude en hiver, pour se protéger du froid.

Dans la dernière période, où ils cohabitent, Hugo continue de se ménager un espace de travail dans les appartements de Juliette. Au 21, rue de Clichy, il travaille à son étage (le second), dans une chambre dont Georges Hugo (le petit-fils), se souvient qu'il l' «appelait son cafarnaüm». C'était une «petite chambre triste, encombrée de brochures, de piles de livres écroulés formant tas de pierres contre les murs; sur une table, les immenses feuilles de "Whatman" couvertes de son écriture, et, près de la fenêtre, le bureau, très haut, où il écrivait debout ${ }^{7} »$. Juliette en décrit le fatras : «[... j’ai mis de côté tes lettres et tes journaux qui commencent déjà à fortement encombrer ma pauvre petite chambre, tellement que je ne sais plus où reposer ma... brosse à tête ${ }^{8}$.» Hugo y travaille le matin, avant sa promenade en omnibus. En son absence, il arrive à Juliette de se risquer à ranger cette pièce qu'elle n'est pas la seule à fréquenter : amoureux fervent et savant austère, Hugo y admet aussi la compagnie du chat Gavroche, au point de l'y enfermer un jour par mégarde9.

\section{Juliette documentaliste}

Dès les premières années, un coin de son logis est déjà réservé aux affaires de travail de Hugo. Dans une armoire, elle conserve des œuvres et des journaux sur lesquels il effectue recherches documentaires et prises de notes; parfois même, elle lui dispose à l'avance les livres dont il aura besoin. Ainsi, début mars 1841, pour préparer son discours de réception à l'Académie française, il lit chez elle les compilations de précédents discours de réception et les œuvres de son prédécesseur Népomucène Lemercier dont il doit donc faire l'éloge malgré le peu d'estime qu'il a pour lui.
Juliette effectue parfois la recherche documentaire ellemême. Après avoir pris des notes sur Lamennais le 10 février 1847, elle avertit Hugo de la fin de son travail :

Je viens de faire l'inventaire des faits et gestes de mon hideux et sale compatriote Lamennais. J'en ai rempli trois grandes feuilles. Je ne sais pas ce qu'on pourra faire de cela, mais ce dont je suis sûre c'est qu'on n'en fera jamais un dithyrambe en l'honneur de la propreté et de la générosité du susdit cancre, quelles que soient l'imagination et la bonne volonté qu'on y apporte. Mais en voilà assez sur ce SALOP ${ }^{10}$.

Ces notes n'ont pas été conservées, à notre connaissance. Mais l'année suivante, à la date du 5 août 1848, Hugo dresse ce portrait peu flatteur :

L'abbé de Lamennais, figure de fouine, avec l'œil de l'aigle, cravate de couleur en coton mal nouée, redingote brune usée, vaste pantalon de nankin, trop court, bas bleus, gros souliers. La déclaration de représentant à la boutonnière. Voix si faible qu'on vient se grouper au pied de la tribune pour l'entendre et qu'on l'entend à peine ${ }^{11}$.

Juliette recueille aussi des renseignements susceptibles de servir à Hugo pour de futurs ouvrages. Ainsi, elle cherche dans un livre du poète bretonnant Auguste Brizeux comment on dit «cheval» en breton, parce que Hugo le lui demande :

Je n'ai trouvé que penn-march cap-du-cheval. C'est dommage que je n'aie pas su plus tôt que tu avais besoin de ce mot parce que j'aurais écrit à mes Bretons de me l'envoyer. Si tu y attaches de l'importance, je peux leur écrire pour le demander tout de suite, tu n' as qu'à parler. Au besoin même on m'enverrait une grammaire bretonne et un guide breton dans lequel tu trouverais tous les mots usuels de cette charmante langue ${ }^{12}$.

6. Où elle habite entre 1856 et 1864.

7. Georges Hugo, Mon grand-père, dans Victor Hugo, Euvres complètes, dir. Jean Massin, Paris, Club français du livre [désormais CFL], t. XV-XVI/2, p. 930.

8. Paris, 13 décembre 1875, lundi soir, 6 h. [Heute/Hovasse].

9. Paris, 9 octobre 1875 [Heute/Hovasse].

10. Paris, 10 février 1847 [Sifferlen/N.]. Comme Juliette, Lamennais est d'origine bretonne.

11. Victor Hugo, Choses vues, Le Temps présent III, 1848 ; Euvres complètes, éd. Jacques Seebacher et Guy Rosa, Histoire, Paris, Laffont, coll. «Bouquins », 1987, rééd. 2002, p. 1070.

12. Paris, 2 janvier 1848 [Kieffer/N.]. Ses «Bretons» sont sa sœur Renée, son beau-frère Louis Koch et son neveu [Jean] Louis Koch. 
Cette recherche était-elle destinée à alimenter le chapitre des Misérables sur l'argot, commencé la veille, où Hugo dresse, dans «Racines», toute une liste de mots étrangers ayant nourri ce langage cosmopolite? Impossible à dire. Nulle trace de «march» (cheval en breton) ne s'y trouve en tout cas, et le Tellmarch, personnage de Quatrevingt-Treize est à ce point postérieur qu'on ne saurait dire si cette recherche, à supposer que Juliette l'ait poussée jusqu'au bout, a pu être utile à Hugo.

Il lui a aussi fait rédiger le journal du retour de voyage en 1843, le récit d'un mariage auquel elle assiste en l'église Saint-Germain-l'Auxerrois, ses souvenirs de couvent, le journal de la révolution de février 1848 , celui du coup d'État (dont il reprendra textuellement certains éléments dans Histoire d'un crime), leur visite aux galeries SaintHubert qui exposent comme une curiosité une famille chinoise, le début de l'exil à Jersey, et le verbatim de diverses conversations - Gérard Pouchain a publié tous ces textes ${ }^{13}$. Juliette exprime sa perplexité sur cette tâche qui fait parfois doublon avec les lettres, et dont elle ne perçoit pas toujours l'utilité. Ainsi, après avoir consigné sa description du mariage à Saint-Germain-l'Auxerrois auquel elle a assisté le 22 avril 1847, elle confesse en mesurant son talent à celui du grand homme :

J'ai écrit hier ce que tu m'as demandé. Ce que j'ai vu seulement car je ne suis pas femme à rien inventer. Je ne suis pas capable de leur faire des enfants après la cérémonie NUPTIALE. Richi et vous avez seuls ce talent d'invention et de production, quant à moi je me borne au simple récit ${ }^{14}$.

Juliette est ici trop modeste, car ce texte est bien tourné et manifeste un sens affûté du détail réaliste et des situations. Mais il ne semble pas avoir été réutilisé par Hugo. Même chose pour les souvenirs d'un marin, fils de son amie Mme Rivière, qu'elle fait venir chez elle à deux reprises, le 16 août et le 6 septembre 1845 , afin de prendre en note le récit de voyage chez les «sauvages 15 » de ce «pauvre matelot à peine dégrossi, mais modeste, si ce mot peut s'appliquer à un homme quelconque et en particulier à un apprenti nautonier ${ }^{16}$ ». Juliette extrait de son témoignage «les quelques particularités intéressantes» dignes d'être transmises à Hugo, après mise «au net 17 ».

Quelques jours plus tard, elle signale un renseignement pris par elle, à la demande de Hugo, auprès de son amie
Eulalie, sur l'origine de la Chandeleur. Il sera réutilisé textuellement dans Les Misérables ${ }^{18}$. Dans la même lettre, elle lui indique avoir consigné à son intention ses connaissances sur la manière de fabriquer des boîtes en carton : Hugo a pu les réutiliser pour exposer la technique mise en œuvre par Thénardier dans cet artisanat au chapitre III, VIII, 19 des Misérables écrit dans les mois qui suivirent.

On apprend aussi qu'il fallut trois jours à Juliette (du 7 au 9 septembre 1847) pour rédiger ses souvenirs de couvent, afin de fournir à Hugo des détails vraisemblables sur la vie quotidienne au Petit-Picpus. Ignorant qu'il en demandait autant, à la même époque, à son autre maîtresse Léonie Biard, Juliette honore la commande avec entrain, et la crâne fierté de contribuer tant soit peu au succès du roman et à la gloire de son amant :

Oui, monsieur, oui, j'ai fini mon manuscrit, oui, j’ai la générosité de vous donner le fruit de mon travail, sans hésiter et sans marchander. Faites-vous de la célébrité avec, faites-vousen de la gloire et de la fortune, je ne m'y oppose pas. Je vous le donne. Je ne vous demande même pas en échange un bout de votre corde. Je vous la laisse tout entière ${ }^{19}$.

Tous les moyens sont bons pour l'aider, jusqu'au cadeau qu'elle lui fait de son petit encrier au moment où il achève

13. Juliette Drouet, Souvenirs (1843-1854), éd. Gérard Pouchain, Paris, Éditions des Femmes - Antoinette Fouque, 2006.

14. Paris, 23 avril 1847 [Sifferlen/N.]. Hugo prenait en note les élucubrations de son coiffeur Richi.

15. Paris, 7 septembre 1845 [Stranart/N.].

16. Paris, 17 août 1845 [Stranart/N.].

17. Paris, 7 septembre 1845 [Stranart/N.].

18. Nicole Savy et Guy Rosa ont identifié cet emprunt et le suivant. Juliette écrit le 25 avril 1847 : «C'est bien la purification de la Vierge, elle a lieu le 2 février et voici ce que dit Mathieu Laensberg à ce sujet : que le soleil luise ou hiberne.......... l'ours rentre dans sa caverne, ce qui fait qu'on a pendant six semaines un froid de loup. Tu vois que je n'oublie pas de faire tes commissions » [Nicole Savy]. Sept ou huit mois plus tard, le renseignement est recyclé dans Les Misérables (III, VIII, 2) : «c'était le 2 février, cet antique jour de la Chandeleur dont le soleil traître, précurseur d'un froid de six semaines, a inspiré à Mathieu Laensberg ces deux vers restés justement classiques: "Qu'il luise ou qu'il luiserne, / L'ours rentre en sa caverne" ".

19. Paris, 9 septembre 1847 [Savy]. C'est l'Angély, le bouffon de Louis XIII, qui offre ainsi son épée à Didier, prêt à se battre en duel contre le marquis de Saverny (ce qui risque de lui valoir la potence) : «En échange, écoutez, pour me porter bonheur, / Vous me laisserez prendre un bout de votre corde» (Marion de Lorme, II, 3). 
l'acte I de Ruy Blas : «Vous devriez m'écrire une bonne petite lettre pour la peine que je vous ai donné mon joli petit encrier. Mais vous ne le ferez pas, il n'y a pas de danger, vous êtes bien trop AVARE pour cela ${ }^{20}$.» Et le lendemain : «J'attends avec impatience la page sortie de mon encrier. Ça doit être bien beau, je voudrais déjà la tenir. D'abord parce que ce serait une preuve que tu as fini21.» Elle dit sa hâte que l'acte I soit achevé (il le sera le lendemain) et qu'il l'emmène en excursion pour fêter ça, comme il le lui a promis.

Il arrive aussi que le chercheur découvre dans une lettre de Juliette une source involontaire, restée inconnue, telle l'onomatopée du coup de canon tiré tous les jours à Guernesey au lever du soleil depuis Castle Cornet, entre six et sept heures, qu'elle forge pour saluer son compagnon le 10 janvier 1865 : «Bum... jour, c'est la manière dont le soleil fait son entrée dans ce pays-ci. J'y réponds par la formule : donnez-vous la peine d'entrer pendant que j'achève le gribouillis de mon autre soleil22.» Deux mois et demi plus tard (le 30 mars, date du manuscrit), Hugo recycle cette trouvaille dans Les Travailleurs de la mer: de Déruchette attristée, le narrateur dit qu' " [o]n ne la voyait plus, le matin, au coup de canon du point du jour, faire une révérence et dire au soleil levant : "bum!... jour. Donnez-vous la peine d'entrer" 23 ».

On s'émerveille aussi de découvrir que le poème des Contemplations «Paroles dans l'ombre», où «elle» regrette que son visiteur ait occupé les quelques heures passées chez elle à écrire sans la regarder, est la mise en vers d'une lettre de Juliette reçue la veille. Le 2 novembre 1846, Juliette se plaint :

Songe que je t'ai très peu vu après tout puisque tu as travaillé tout le temps sans lever une seule fois les yeux sur moi et sans m'adresser une parole. Je sais bien que je pouvais te regarder, et je ne m'en suis pas privée, mais je ne te vois pas aussi bien quand tu ne me regardes pas un peu toi-même de temps en temps 24 .

Les «Paroles dans l'ombre» des Contemplations (II, 15) s'achèvent ainsi :

Sans doute, je vous ai; sans doute, je vous voi. La pensée est un vin dont les rêveurs sont ivres, Je le sais ; mais, pourtant, je veux qu'on songe à moi.

Quand vous êtes ainsi tout un soir dans vos livres,
Sans relever la tête et sans me dire un mot,

Une ombre reste au fond de mon cœur qui vous aime; Et, pour que je vous voie entièrement, il faut

Me regarder un peu, de temps en temps, vous-même 25 .

Repérer ce genre d'emprunt est ardu 26 : seule la connaissance livresque du chercheur, appuyée sur la datation des œuvres facilitée notamment par la chronologie Massin en ligne sur le site du groupe Hugo, permet de les identifier. Certains nous ont donc sûrement encore échappé.

\section{Juliette gardienne et copiste des manuscrits}

Autre émotion : entrer dans la confidence du soin jaloux avec lequel Juliette choyait les manuscrits. Elle garde ainsi chez elle le portefeuille du Rhin, et se lamente, le 9 octobre 1844, que Hugo l'ait égaré après l'avoir rapporté chez lui :

Je suis bien fâchée qu'on t'ait pris le portefeuille du Rhin. Outre le dommage que cela te cause, c'est une perte irréparable pour moi et qui me fait un très vif chagrin. Vois-tu, mon Toto, tu ne devrais jamais rien laisser traîner de ton écriture chez toi. Et tu devrais ne jamais me rien reprendre de ce qui m'appartient si légitimement. Tu en vois les inconvénients 27.

Dès le départ de Hugo en exil, le 11 décembre 1851 au soir, Juliette, comme l'explique Jean-Marc Hovasse, «veille comme à la prunelle de ses yeux sur la vieille malle aux

20. Paris, 12 juillet 1838 [Sandra Glatigny/Pouchain].

21. Paris, 13 juillet 1838 [Glatigny/Pouchain].

22. Guernesey, 10 janvier 1865 [Stranart/N.].

23. Les Travailleurs de la mer, III, I, 1. C'est Jeanne Stranart et Gérard Pouchain qui ont fait cette découverte.

24. Paris, 2 novembre 1846 [N.].

25. Voir F. Naugrette, «Une lettre de Juliette Drouet, source du poème de Victor Hugo "Paroles dans l'ombre" ", Revue d'Histoire littéraire de la France, «Correspondances d'écrivains et histoire littéraire», 2012-4, p. 949-954.

26. Des similitudes entre des expressions employées par Juliette et des formules de la Tisbe dans Angelo tyran de Padoue, de Marcinelle et Eurydice dans L'Intervention, ont été repérées par Anne Ubersfeld dans Le Roi et le Bouffon (Paris, Corti, 1974, p. 261-262), et par Arnaud Laster dans «Quand les personnages du théâtre de Victor Hugo parlent comme Juliette», dans Juliette Drouet. Mon âme à ton coeur s'est donnée, Paris, Maison de Victor Hugo/Paris Musées, 2006, p. 43-47.

27. Paris, 9 octobre 1844 [Caroline Lucas/N.]. 
manuscrits 28 » qu'elle apporte avec elle à Bruxelles où elle le rejoint deux jours plus tard. En mars 1852, quand la rédaction d'Histoire d'un crime fait planer le risque d'un deuxième exil, elle lance un avertissement : la malle «ne pourrait pas supporter un second voyage tant elle est détraquée». Il faut en acheter une neuve, «la meilleure et la plus solide 29 » qu'il puisse trouver. Elle s'occupe aussi de protéger l'œuvre des regards indiscrets :

J'ai serré votre manuscrit sous clef ce matin. Maintenant je ne veux pas qu'il traîne sur ma table. Tu devrais de ton côté, mon Victor, n'en pas laisser une seule ligne dehors, cela devient de plus en plus prudent. Quant à moi je te promets de faire bonne garde. [...] Personne mieux que toi ne peut prévoir toutes les embûches et toutes les trahisons. Aussi je te supplie de ne rien épargner pour les déjouer toutes. Pour cela il suffit d'un peu de soin et d'attention de ta part 30 .

Ce soin et cette attention, Juliette craint souvent que Hugo n'en manque, comme lorsqu' elle lui recommande en 1878 de faire l'inventaire de ses armoires guernesiaises :

De ton côté, tu ferais peut-être bien de vaincre ta paresse ou ton indifférence, toutes les deux à la fois, probablement, pour faire une revue de tes armoires, dites profondes. Tu y retrouverais peut-être les trois actes manuscrits de LES JUMEAUX et beaucoup d'autres choses encore avec 31 .

Cette mise en garde semble indiquer que Hugo n'avait pas rapatrié tous ses manuscrits lors de son rapide séjour à Guernesey en avril 1875, et que sa belle-sœur Julie Chenay, qui entretenait Hauteville House en son absence, en avait encore quelques-uns sous sa protection plus ou moins directe.

L'autre soin que Juliette prend des manuscrits de Hugo, c'est leur copie. Un de ses nombreux autoportraits, «l'esquisse d'une femme appliquée à faire des alinéas ${ }^{32}$ », la représente le 7 août 1841 reproduisant à la plume qu'elle vient de tremper dans son encrier une page de Hugo (fig. 1). Il l'emploie à reproduire tel carnet ${ }^{33}$, ou telle lettre dont il veut conserver le double en tout ou partie. Le compte rendu de cette activité délivre indirectement des indications utiles au chercheur, tel cet anodin «Je viens de copier l'extrait de ta lettre à Janin ${ }^{34}$ », qui permet de corriger l'attribution pour le moins conjecturale à Hetzel, dans l'édition du Club français du livre, de cette lettre au destinataire jusqu'à présent

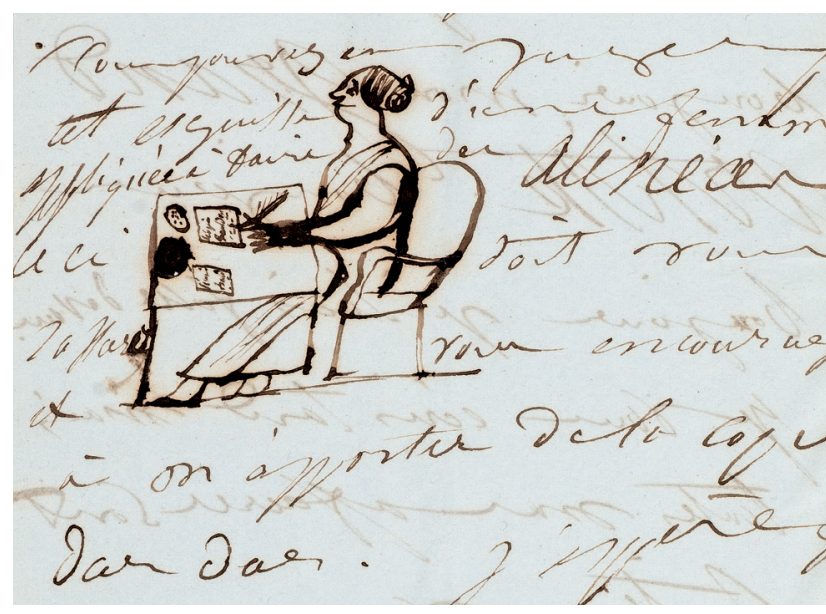

Fig. 1 : Détail de la lettre du 7 août 1841 (BnF, Naf 16346, f. 123-124)

inconnu 35 . Hugo lui confie aussi, dès septembre 183436 , la copie de ses œuvres, activité délicieuse, parce qu'il lui accorde ainsi non seulement la primeur de leur découverte, mais aussi une confiance inestimable dans sa discrétion et sa compétence. Copier, pour Juliette, c'est, trop rarement, enfin et tant soit peu se rendre utile, soigner son malheur de lui être à charge.

28. J.-M. Hovasse, Victor Hugo, t. II, Pendant l'exil I, 1851-1864, Paris, Fayard, 2008, p. 11.

29. Bruxelles, 7 mars 1852 [Duthion/N.].

30. Bruxelles, 24 mars 1852 [Duthion/N.].

31. Guernesey, 27 octobre 1878 [Pouchain].

32. Paris, 7 août 1841 [Sifferlen/N.].

33. C'est ce qu'elle indique dans une lettre de 1834 que l'on peut dater du 3 novembre [Stranart/N.].

34. Jersey, 22 avril 1855 [Magali Vaugier/Rosa].

35 . Lettre du 22 avril 1855, qui n'avait au demeurant pas non plus été reprise en 2004 par Sheila Gaudon dans son édition de la correspondance entre Victor Hugo et Pierre-Jules Hetzel; CFL, t. IX, p. 1089.

36. «Il n'est pas tout à fait $6 \mathrm{~h}$. du soir, je viens de finir de copier les vers que tu m'as donnés hier. Je ne suis pas très familiarisée avec les compliments en usage dans le beau monde. Tout ce que je puis te dire, c'est que j'ai pleuré et admiré en t'entendant les lire, c'est que j'ai pleuré et admiré en les relisant, c'est que je pleure et que j'admire en me les rappelant. Je te remercie du fond de l'âme d'avoir pensé à moi en les faisant » [Stranart/N.]. La veille, Hugo a glissé pour elle dans un châtaignier de la forêt des Metz le poème «Oh! pour remplir de moi ta rêveuse pensée » qui deviendra la pièce XXIV des Chants du crépuscule. 
Preuve qu'elle nourrit pour les manuscrits de Hugo un amour fétichiste, la jalousie qu'elle exprime à leur égard est aussi farouche que s'il s'agissait de prêter Hugo lui-même à une autre. D'autant que les copistes que Hugo lui adjoint sont le plus souvent des femmes : Léopoldine, la jeune Adèle, et à l'époque de la reprise des Misérables pendant l'exil Victoire Étasse, la petite Bénézit et Julie Chenay (la jeune sœur de Mme Hugo); sur la fin de sa vie, c'est à son secrétaire bénévole Richard Lesclide que revient cette prérogative. Aussi Juliette est-elle aux anges lorsque Hugo lui confie encore de la copie malgré sa mauvaise vue :

J'espère, mon grand adoré, que, si tu as quelques nouvelles merveilles à faire copier de Toute la lyre, tu me favoriseras en m'en confiant l'honneur et le bonheur en même temps qu'au bon Lesclide qui sera si heureux que tu lui continues cette marque de confiance qu'il a religieusement méritée jusqu'à présent. Quant à moi, [...] je te promets d'y mettre tout mon zèle, à défaut de belle écriture, et tout mon cœur à défaut d'esprit ${ }^{37}$.

L'expression de son souci de bien faire permet au chercheur de glaner d'intéressants détails. Le 13 octobre 1845, elle supplie Hugo, même s'il trouve qu'elle ne travaille «pas assez vite ${ }^{38}$ » de ne pas acheter de presse à copier. Pendant qu'elle copie Les Misérables, elle fait « songer [Hugo] à [lui] apporter du papier à copire puisque [il] tien[t] à ce qu'il soit pareil d'un bout à l'autre ${ }^{39}$ »; après s'être fait reprendre, avec Julie Chenay, sur des erreurs de ponctuation, elle proteste de son sérieux :

[...] je suis impatiente de savoir comment tu trouveras notre besogne actuelle à Mme Chenay et à moi. [...] Cependant j'ai la conscience que je m'applique à n'avoir aucune distraction et à ne rien laisser passer mais cela ne suffit pas pour me rassurer et je ne serai vraiment tranquille que lorsque tu auras donné toi-même ton satisfecit. En attendant je suis assez mal dans mes : point et virgule, et je souffre sans exclamation et je surveille la pauvre petite Mme Chenay sans pitié à la ligne et je t'aime au milieu de tout cela sans ponctuation 40 .

Ce qui confirme, au stade de la copie, ce que l'on savait déjà sur un des problèmes rencontrés par Hugo avec ses éditeurs au stade des épreuves : il doit vérifier de près qu'on ne dénature pas sa ponctuation ${ }^{41}$.
Juliette et Julie Chenay sont de nouveau sollicitées pour l'aider à la collation, au moment des épreuves : elles comparent celles-ci à l'original, et dressent le relevé des corrections faites par Hugo. Mais elles n'effectuent pas elles-mêmes les corrections, contrairement à ce que Hugo fait croire par plaisanterie à leur ami Kesler, au grand dam de Juliette :

Pourquoi donc as-tu mythifié hier le gros Quesnard en lui faisant croire que je CORRIGEAIS tes épreuves? Ne crainstu pas qu'en disant de telles invraisemblances tu ne fasses ressortir davantage encore mon ignorance flagrante et qu'un ridicule indélébile ne s'attache à moi, pauvre femme qui n'en peut mais de tes plaisanteries hyperboliques 42 ?

Le compte rendu de son activité de lectrice/copiste permet d'affiner des datations pour des phases de rédaction, notamment pour la composition de certaines lettres du Rhin ${ }^{43}$. De même, la citation approximative du poème «Lacrymae rerum» des Voix intérieures dans une lettre du 6 mai 1837, alors que le manuscrit porte la date finale du 15 mai, donne une indication sur la date du début de sa rédaction ${ }^{44}$. De même encore, la mention, dans la lettre du 18 juin 1852, de «la plus grande attention» et «la plus grande admiration» avec lesquelles elle vient de lire ses «7500000 voix» permet de dater au moins une partie de la rédaction de ce qui deviendra le livre sixième de Napoléon le Petit. Enfin, alors qu'on croyait jusqu'à présent que Hugo avait mis la dernière main à son discours de réception à l'Académie française le 16 avril 1841, la lettre du surlendemain permet de rectifier cette date :

Vous avez cependant fini votre discours hier, et vous m'aviez bien promis de signaler cet heureux événement par un

37. Paris, 19 novembre 1878 [Chantal Brière].

38. Paris, 13 octobre 1845 [Stranart/N.].

39. Guernesey, 12 février 1861 [Sophie Gondolle/N.]. «Copire» est un néologisme récurrent pour «copier».

40. Guernesey, 28 décembre 1861 [Naugrette].

41. Voir Jacques Dürrenmatt, «Virgules et blancs : une question d'importance? », Hugo et la langue, dir. F. Naugrette et G. Rosa, Rosny-sousBois, Bréal, 2005.

42. Guernesey, 23 février 1862 [Marie Rouat/Brière]. Quesnard est le surnom qu'ils donnent à Kesler.

43. La thèse de G. Sifferlen, éditrice des lettres de 1841, apporte sur ces datations de nouvelles précisions.

44. Juliette cite «Son grand casque doré sur sa tête de pierre. Ou à peu près» [Sylviane Robardey-Eppstein]. 
déjeuner sterling et par la lecture du susdit le soir même. Mais vous promettez toujours plus de beurre que de pain. Taisezvous, taisez-vous, vous êtes un académicien 45 .

Lorsque Juliette souligne comme ici le mot «déjeuner», elle entend par là d'autres plaisirs des sens que le seul repas (quant à «sterling», le mot employé par elle comme adjectif signifie «important, de taille, considérable»). Si cette date peut être aujourd'hui rectifiée, on le doit donc au dépit amoureux de Juliette, à qui Hugo n'a pas tenu sa promesse galante.

\section{Témoignages de lectrice et d'auditrice}

Qu'elle copie, lise ou entende Hugo lui dire ses œuvres, elle exprime systématiquement son admiration. À quoi s'ajoute une infinie gratitude lorsqu'elle reçoit des poèmes qui lui sont dédiés, ce qui permet au chercheur de confirmer l'identité de leur muse ou dédicataire anonyme. C'est le cas de «À Ol. » qui deviendra le poème XII des Voix intérieures, écrit le 26 mai 1837, et dont elle le remercie le lendemain :

Merci de me consacrer quelques vers de ta poésie. Moi je te donne ma vie, mon âme à tout jamais. Et j'ai la douceur de penser qu'avec cela je suis aussi généreuse que toi. [...] En échange de vos admirables vers, je vous donne mon amour, sous toutes les formes et sous toutes les espèces. Je n'ai pas autre chose à vous donner. C'est mon esprit à moi. C'est ma poésie 46 .

Même chose pour «À cette terre où l'on ploie», qui figurera dans Les Rayons et les Ombres, qu'elle cite juste après l'avoir reçu pour la Sainte-Julie, le 21 mai 1838 :

J'ai baisé toutes les lettres de tous les mots adorables de ton écriture bien aimée. Je n'essayerai pas de te dire mon ravissement en lisant ta chère petite lettre et tes admirables beaux vers 47 .

Elle commente spontanément ce qu'il lui donne à lire ou copier. Le tiers-lecteur peut bien sourire à la lecture de ses commentaires littéraires, ils n'en sont pas moins judicieux et souvent pleins d'esprit, comme lorsqu'elle se moque de son penchant à l'affabulation dans le récit de son voyage au Rhin :

Vous pensez bien, mon amour, que pour ne vous avoir pas copié tout de suite je n'ai pas eu la stupide patience d'attendre à demain pour vous dévorer : paysages, enfant, houris, chaises de poste, tonnerre, voyageurs, bateau-poste, cimetière, sérénade ours, ours, ours, ours, ours, ours, etc... le loup, la masure, la vieille femme, l'église, baraque en plein vent, villageois, villageoises, égyptien, Sbrigani, philosophes de l'Antiquité, poètes, gale, chameau, Hurons, Botocudos, Mittigouchiouekendalakiank, charlatan et académicien. [...] Quel homme vous êtes! Quelle platine que votre crayon ou votre plume! Quelle cascade de menteries, quelle effroyable avalanche de blagues, quelle consommation d'ours. Saint Antoine lui-même, le petit et le grand saint Antoine, pâliraient devant cette magnifique guirlande d'ours tressée pour la plus grande admiration et la plus parfaite crédulité des six mille milliards de lecteurs qui la goberont en lisant cela 48 .

De même, ses commentaires sur la genèse des Misérables qu'elle suit au rythme de leur copie rendent compte bien avant la publication du roman de sa structure haletante et de l'empathie qu'il suscite chez le lecteur :

Je suis très pressée de continuer mon TRAVAIL. Cette activité a plus d'une raison dont la première est très sincèrement l'ardente curiosité que j'ai de savoir ce que deviennent tous ces pauvres martyrs de votre imagination. Je m'y intéresse comme des personnages de vrai et qui me toucheraient de très près. Leurs malheurs me rendent très malheureuse et je voudrais griffer ce monstre de Javert et tuer ces hideux Thénardier. [...] Toutes ces sublimes choses me font désirer de tout savoir tout de suite et je voudrais ne m'arrêter qu'au mot : fin pour connaître le sort que tu réserves à tous ces pauvres gens. D'abord si tu ne rends pas les Thénardier les plus malheureux et les plus immondes des gredins tu ne seras pas juste. Je veux pour ma part que tu leur fasses tout le mal possible ou je ne serai pas contente. Je ne suis pas généreuse comme tu sais. D'ailleurs il n'y a pas de générosité qui puisse faire pardonner à de pareils monstres 49 .

Spectatrice de mélodrames, lectrice de romans-feuilletons, Juliette lit la fiction avec l'horizon d'attente propre à ces

45. Paris, 18 avril 1841 [Sifferlen/N.].

46. Paris, 27 mai 1837 [Robardey-Eppstein].

47. Paris, 21 mai 1838 [Hélène Hôte/N.].

48. Paris, 14 juillet 1841 [Sifferlen/N.]. Paraphrase d'un passage de la lettre vingtième du Rhin.

49. 4 janvier 1848 [Kieffer/Michèle Bertaux]. 
genres populaires, tout en sachant que Hugo en joue, et le déjoue :

Je suis impatiente de retrouver mon pauvre Jean Tréjean et la pauvre petite Cosette. J'avoue que leur sort me tient à cœur et que je voudrais à tout prix les voir heureux. Je ne sais pas si cela dépend de toi mais dans tous les cas il est impossible de plus s'identifier avec les malheurs de ces pauvres gens que je ne le fais 50 .

Juliette a compris que les fins de Hugo ignorent la providence, qu'inversement il laisse toujours une chance au méchant, et que le sort des personnages dépend de la toutepuissance de l'auteur, non pas de Dieu.

Parfois Hugo lui fait lui-même la lecture à haute voix. Juliette en raffole, et s'en montre jalouse. Ainsi, en décembre 1847, elle enrage que son amant rapporte chez lui un soir le manuscrit de Jean Tréjean qu'il laissait jusqu'alors chez elle, et fasse profiter sa famille d'une lecture publique :

J'en étais bien sûre, que vous ne reviendriez pas une fois que vous auriez emporté ce cher manuscrit. Avec ça que vous pourrez bien tout lire aujourd'hui. Quand je pense que vous ne trouvez pas le temps de m'en lire à moi une pauvre petite page ou deux, je suis furieuse. Laissez faire, allez, quand j'aurai des manuscrits à moi je ne vous les montrerai pas et ils vous passeront devant le nez un peu gentiment. Il paraît que mon admiration à moi ne vaut pas celle des autres? Malhonnête, on vous en fichera des Juju littéraires comme moi pour avoir l'air de cracher dessus. [...] Vous êtes libre, seulement je vous défends à l'avenir de me faire corriger vos pataquès : gardez-les pour vos intimes et pour vos préférées 51 .

Ayant continué de réclamer son dû les jours suivants, elle sera exaucée le 23 décembre :

Je ne sais comment t'exprimer tout ce que j'ai éprouvé de poignantes douleurs et d'admiration passionnée pendant cette prodigieuse lecture. Il me semblait [...] que tu emportais mon âme à travers les terribles mondes de ton génie. Ma pensée suivait ta parole et tout ce que j'ai d'intelligence, de cœur et d'âme admirait, souffrait et priait selon que tes sublimes paroles tombaient de tes lèvres dans mon esprit. Ces choseslà ne se racontent pas, on les sent 52 .

Son témoignage, souvent concordant avec les carnets et agendas de Hugo, montre la fréquence de ces lectures à haute voix pour le cercle des intimes (où elle finit par être admise). Ainsi, à Guernesey, pendant le séjour de Paul Meurice, c'est «tous les jours 53 », du 22 au 27 juin 1873, qu'il entreprend de leur lire Quatrevingt-Treize, récemment achevé, «d'un bout à l'autre 54 ».

\section{Relations de Hugo avec ses éditeurs, ses interprètes et ses directeurs de théâtre}

Juliette suivant toutes les étapes de la création des œuvres, elle donne aussi son avis sur leur fabrication et leur promotion. Bien plus que les périodes d'écriture et de relecture, Juliette redoute les mois et semaines précédant la publication : Hugo est si absorbé par les démarches avec les éditeurs qu'il a à peine le temps de lui rendre visite. Frustrée de la présence de son homme avant la sortie des Voix intérieures (fin juin 1837), elle se morfond dans sa solitude : «Je crains même de rester dans cet état jusqu'à la parfaite publication de votre beau livre [...]. Il est vrai que vous m'avez promis de vous occuper immédiatement et sans désemparer d'une pièce, ce qui fera un été complet55. » À cette date, il est vraisemblable (mais pas certain) qu'il s'agit du projet de Ruy Blas, dont Anne Ubersfeld a montré les différentes étapes de la lente germination depuis 1835, dont un brouillon qu'elle date de l'automne 1836, écrit sur le papier qu'il fournit à Juliette à cette époque 56 . Ce projet (ou un autre) est ensuite évoqué de nouveau les 23 juillet et 27 septembre 1837, mais ce dernier jour Juliette regrette qu'il n'y ait aucun rôle pour elle dans la future pièce. Faut-il comprendre qu'il ne s'agit pas de Ruy Blas, puisqu'elle croira devoir y jouer la Reine? Ou bien qu'il s'agit déjà de Ruy Blas, dont Hugo lui fera miroiter le grand rôle féminin ultérieurement 57 ?

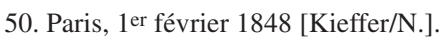

51. Paris, 17 décembre 1847 [Savy].

52. Paris, 24 décembre 1847 [Savy].

53. Guernesey, 26 juin 1873 [Maggy Lecomte et Manon Da Costa/N.]

54. Guernesey, 28 juin 1873 [Lecomte et Da Costa/N.].

55. 31 mai 1837 [Sylviane Robardey-Eppstein].

56. Anne Ubersfeld, édition critique de Ruy Blas, Paris, Les Belles Lettres, 1971, p. 26.

57. Comme le suggère $S$. Robardey-Eppstein, «s'il s'agit ici d'une autre pièce que Ruy Blas projetée par Hugo, seuls les fragments dramatiques de la période pourraient apporter un début de réponse pour l'identifier », 2e note de son édition de la 2e lettre du 27 septembre 1837. 
La préparation de Châtiments cause bien du souci à Hugo, qui l'avoue à Louise Colet : «Je fais en ce moment une œuvre de titan : ce n'est pas d'écrire un livre contre un homme, c'est de le publier58.» On suit avec Juliette les atermoiements des éditeurs belges : elle réconforte Hugo après la défection de Tarride, refroidi par la contraignante et répressive loi Faider : «Je ne veux pas que tu sois triste, ni même contrarié, de l'incident belgiquois. J'ai le pressentiment que tu trouveras une combinaison meilleure que celle de Tarride 59 . » Puis elle suit au jour le jour la correspondance avec Hetzel et, après la signature du contrat final avec Henri Samuel, s'inquiète lorsque le mauvais temps, interrompant la liaison entre la Belgique et Saint-Hélier, retarde la livraison des épreuves ${ }^{60}$. Les difficultés de Hugo avec ses éditeurs belges, recensées par Jean-Marc Hovasse ${ }^{61}$, la tracassent : à plusieurs reprises, elle se plaint de ces «lambins d'éditeurs belges 62 » qui retardent la publication des Misérables.

Elle le met en garde contre les directeurs de théâtre prêts à l'escroquer sur ses droits d'auteur. Elle craint ainsi, le 29 novembre 1838, que le mauvais temps ne fournisse un nouvel argument pour « la comédie que Villeneuve essaye de jouer avec les recettes de Ruy Blas ${ }^{63}$ ». Trois ans plus tard, pour la reprise à la Porte-Saint-Martin, elle veille encore au grain :

Il paraît que très décidément vous abandonnez l'infortuné Ruy Blas à lui-même ? C'est encore très spirituel, ça, et digne de votre magnifique indifférence à l'endroit de vos droits d'auteur. Vous avez bien raison, l'or est une chimère, sachons nous brosser le ventre au soleil 64 .

Selon elle, il faut assister le plus souvent possible au spectacle - ce que Hugo néglige alors -, pour surveiller les directeurs, «les contenir et les empêcher de faire de certaines perfidies 65 », «encourager» et «stimuler» les acteurs qui, si on leur laisse la bride sur le cou, dénaturent leurs rôles par cabotinage ou négligence, tel Frédérick Lemaître qui, à l'en croire, dans une reprise de Ruy Blas à la Gaîté en 1850, a «plutôt bredouillé que parlé, [...] plutôt éteint qu'illuminé $66_{»}$ son rôle. Aussi ne se plaint-elle pas trop quand Hugo prend soin d'assister à un maximum de répétitions, comme le mois qui précède la reprise de Marion de Lorme à la Comédie-Française en mars 1838 :

Sans doute tu es à la répétition? Jusqu'à ce que Marion soit donnée, je ne pourrai plus compter sur toi le matin? Je ne t'en veux pas, bien au contraire, pauvre âme. [...] Je hâte de tous mes vœux la fin des répétitions pour que nous soyons un peu plus l'un à l'autre, et puis pour être sûre que la rage des comédiens qui ne jouent pas dans cette pièce a été impuissante devant le succès 67 .

Après le retour d'exil, elle l'encourage à faire répéter lui-même ses acteurs. Lafontaine, dont le cabotinage est proportionnel à la sottise, vient ainsi répéter Ruy Blas à domicile le 16 janvier 1872. Mais lorsqu'en 1877 Hugo assiste sans elle aux répétitions d'Hernani à la ComédieFrançaise, elle ne peut dissimuler sa jalousie et le supplie de ne pas s'attarder "plus que de raison dans cette Capoue de carton et de maquillage 68 ».

\section{Pour en finir... avec Les Burgraves}

Juliette va voir les pièces de Hugo aussi souvent que possible. Son témoignage du lendemain matin fournit des renseignements de première main sur le jeu des acteurs et sur l'accueil du public. Les jugements qu'elle porte sur ses anciens camarades doivent cependant être pris avec précaution : la jalousie et la rancœur s'y mêlent parfois. Jamais un compliment sur Mlle George, qui en 1833 a contribué à sa chute. Elle l'appelle «la vieille femme», «la grosse dame», et l'utilise comme comparaison comique : «Toi tu es une grosse bête, plus grosse que l'éléphant et que Mlle George69». Jamais un compliment non plus sur

58. Victor Hugo à Louise Colet, 28 juin 1853 ; CFL, t. VIII, p. 1071.

59. Bruxelles, 2 mars 1853 [Duthion/N.].

60. Jersey, $1{ }^{\mathrm{er}}$ septembre 1853 [Duthion/N.].

61. Sur cette question, voir J.-M. Hovasse, Victor Hugo chez les Belges, Bruxelles, Éditions Le Cri, 1994. Voir aussi sa biographie de Hugo, op. cit., t. II.

62. Guernesey, 8 mars 1862 [Heute/N.].

63. Paris, 29 octobre 1838 [Élise Capéran/N.]. Ferdinand de Villeneuve dirige alors, avec Anténor Joly, le Théâtre de la Renaissance qui vient d'être inauguré avec Ruy Blas.

64. 21 août 1841 [Sifferlen/N.]

65. Paris, 18 août 1841 [Sifferlen/N.].

66. 28 juillet 1850 [Kieffer/N.].

67. Paris, 13 février 1838 [Marie Rouat/N.].

68. 18 novembre 1877 [Rosa].

69. Paris, lettre non datée de 1834, BnF, Naf 16322, f. 251-252 [Stranart/N.]. 
Frédérick Lemaitre qu'elle met au rang des «affreux histrions $70_{\gg}$ : elle ne lui pardonnera jamais d'avoir été dépossédée du rôle de la reine de Ruy Blas en 1838 au profit de sa maîtresse Louise Beaudouin.

En revanche, on peut se fier à son témoignage sur l'accueil du public : elle mentionne ses variations soir après soir, enchantée des bravos, ulcérée par les sifflets. Aussi n'y a-t-il pas lieu de remettre sa parole en doute lorsqu'elle témoigne, après chaque représentation des Burgraves au printemps 1843, de l'accueil contrasté du public. Le lendemain de la première, elle se réjouit de «la victoire d'hier malgré la malveillance évidente qu'il y avait dans la salle» (7 mars); les jours suivants, tantôt elle enrage, rentrant chez elle «furieuse et indignée» (16 mars) ou «malade de colère» (25 mars), tantôt elle exulte après «une des plus belles soirées que tu aies jamais eues» (10 mars), une «magnifique soirée» (22 mars $\left.{ }^{71}\right)$, ne sachant jamais à quoi s'attendre : «Jusqu'à présent les représentations bonnes et mauvaises ont tellement alterné que je n'ose pas me fier d'avance à celle de ce soir» (25 mars). En réalité, la cabale fomentée par Mlle Maxime n'a pas réussi à faire chuter la pièce 72 , ce qu'accrédite aussi sa reprise à la Comédie-Française en 1846 : on ne reprenait pas les œuvres qui avaient chuté à la création. Le témoignage de Juliette corrobore donc les études de réception sur cette mystification du prétendu «échec » des Burgraves 73 , et invite à renoncer enfin à cette « contre-vérité officielle de l'histoire littéraire ${ }^{74}$ ».
Le journal épistolaire de Juliette a encore beaucoup à nous apprendre sur les habitudes de travail de Hugo, ses sources, la chronologie de son œuvre, la manière dont il corrigeait ses manuscrits et ses épreuves, ses relations avec ses éditeurs, les directeurs de théâtre et les comédiens, la réception de son théâtre. Non seulement il éclaire d'un jour nouveau la genèse des œuvres de Hugo, mais il invite aussi à réviser certaines légendes de l'histoire littéraire : nous ne sommes pas au bout des révélations que nous réservent les milliers de pages sorties de son encrier.

70. Paris, 22 janvier 1843 [Paploray/N.].

71. Les dates sont celles des représentations, dont elle témoigne dans ses lettres du lendemain [Paploray/N.].

72. Ces conclusions proviennent du mémoire de master [2009] d'Olivia Paploray, éditrice de l'année 1843.

73. Voir Patrick Berthier, «L'"échec” des Burgraves», Revue d'Histoire du Théâtre, no 187, 1995; Olivier Bara, «Le triomphe de la Lucrèce de Ponsard (1843) et la mort annoncée du drame romantique : construction médiatique d'un événement théâtral», dans Qu'est-ce qu'un événement littéraire au XIXe siècle ?, dir. Corinne Saminadayar-Perrin, Saint-Étienne, Publications de l'Université de Saint-Etienne, 2008.

74. Pierre Laforgue, «La division séculaire dans l'histoire de la littérature », Histoires littéraires, no 9, 2002, p. 25. 
Florence NaUgrette, professeur à l'université Paris-Sorbonne, est l'auteur des essais Le Théâtre romantique. Histoire, écriture, mise en scène (Seuil, 2001), Le Plaisir du spectateur de théâtre (Bréal, 2002), Le Théâtre de Victor Hugo (Ides et Calendes, 2016), et le coauteur de l'ouvrage Le Théâtre français du XIXe siècle (L'Avant-Scène, 2008). L'équipe de chercheurs qu'elle dirige publie l'édition en ligne du journal épistolaire (22000 lettres) adressé par Juliette Drouet à Victor Hugo.

florence.naugrette@orange.fr

\section{"La page sortie de mon encrier»}

Le journal épistolaire de Juliette Drouet, en cours d'édition sur le site en accès libre < www.juliettedrouet.org >, présentera à terme les quelque 22000 lettres envoyées par Juliette à Hugo pendant un demi-siècle. Cette masse de documents, inédits à $90 \%$, éclaire plusieurs aspects de la genèse de l'œuvre de Victor Hugo, à commencer par des aperçus directs sur ses habitudes de travail (rythme et méthode). Juliette Drouet aide Victor Hugo non seulement en l'inspirant mais en lui servant aussi épisodiquement de documentaliste. Elle veille en retour avec un soin jaloux sur ses manuscrits qu'elle lit, écoute et recopie pour lui, ce qui est pour elle une façon de se les approprier. Ses témoignages de premier plan permettent de corroborer, ou de rectifier, sur plus d'un point la chronologie de la rédaction, mais aussi de suivre la genèse éditoriale, et la réception des œuvres, notamment théâtrales.

The publication of Juliette Drouet's epistolary diary, in progress on the free access website <www.juliettedrouet.org $>$, will eventually present the 22000 letters Juliet sent Hugo during half a century. This mass of documents, $90 \%$ unpublished, sheds light on several aspects of the genesis of Victor Hugo's work, starting with direct insights on his work habits (rhythm and method). Juliette Drouet helped Victor Hugo not only by inspiring him but also by occasionally assisting him with research. She very carefully watched over his manuscripts that she read, listened to and copied for him, which was a way for her to appropriate them. Her important first hand testimony enables us to corroborate or correct many details of the writing's chronology, but also to follow the editorial genesis and the works' reception, in particular for the plays.

Das Brieftagebuch von Juliette Drouet, dessen Edition auf der

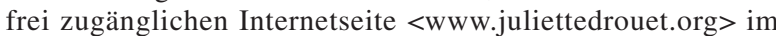
Entstehen ist, wird den Lesern nach Fertigstellung 22.000 Briefe zugänglich machen, die Juliette im Laufe eines halben Jahrhunderts an Victor Hugo schrieb. Diese große Menge an Dokumenten, die zu 90\% noch nicht ediert wurden, erhellt mehrere Aspekte der Genese von Hugos Werk. Dazu gehören direkte Einblicke in seine Arbeitsgewohnheiten (Rhythmus und Methode seiner Arbeit). Juliette Drouet hilft Victor Hugo nicht nur, indem sie ihn inspiriert, sondern zeitweise auch als Dokumentalistin. Sie wacht mit eifersüchtiger Sorge über seine Manuskripte, die sie liest, sich vorlesen lässt und für ihn abschreibt, was für sie eine Methode der Aneignung darstellt. Ihre bedeutenden Zeugnisse erlauben an mehreren Stellen, die Chronologie der Redaktion zu bestätigen oder zu berichtigen, aber auch die Genese der Edition und die Rezeption der Werke - besonders der dramatischen - zu verfolgen.
E1 diario epistolar de Juliette Drouet, que está siendo editado en el sitio de acceso libre <www.juliettedrouet.org $>$, presentará, con el tiempo, las casi 22000 cartas enviadas por Juliette a Hugo durante medio siglo. Esta masa de documentos -inédita en un 90\% - esclarece varios aspectos de la génesis de la obra de Víctor Hugo, empezando por varios detalles observados de sus hábitos de trabajo (ritmo y método). Juliette Drouet ayuda a Víctor Hugo, no sólo inspirándolo, sino también sirviéndole episódicamente de documentalista. En contrapartida, se ocupa de sus manuscritos con una solicitud exclusiva: los lee, los escucha, se los copia, lo que representa para ella una manera de apropiárselos. Sus testimonios, de gran importancia, permiten corroborar, o rectificar, en más de un punto, la cronología de la redacción y seguir la génesis editorial y la recepción de las obras, en particular las teatrales.

O diário epistolar de Juliette Drouet, em edição no sítio de livre acesso <www.juliettedrouet.org>, apresentará a seu tempo as cerca de 22000 cartas enviadas por Juliette a Hugo durante meio século. Essa massa de documentos, de que estão inéditos uns $90 \%$, ilumina vários aspectos da génese da obra de Victor Hugo, a começar por relances directos sobre os seus hábitos de trabalho (ritmo e método). Juliette Drouet ajuda Victor Hugo não só como fonte de inspiração, mas também episodicamente como documentalista. Ela monta uma guarda ciosa sobre os manuscritos que lê, escuta e recopia, e de que assim se apropria. O seu testemunho de primeira mão permite confirmar, ou corrigir, em mais de um ponto a cronologia da escrita, acompanhando a génese editorial e a recepção das obras, nomeadamente teatrais.

I 1 diario epistolare di Juliette Drouet, in corso di pubblicazione sul sito ad accesso libero www.juliettedrouet.org, presenterà a termine le quasi 22.000 lettere inviate da Juliette à Hugo nel corso di mezzo secolo. Questa massa di documenti, inediti al $90 \%$, chiarisce molti aspetti della genesi dell'opera di Victor Hugo, a cominciare da testimonianze dirette sulle sue abitudini di lavoro (ritmo e metodo). Juliette Drouet aiuta Victor Hugo non solo ispirandolo ma facendogli occasionalmente anche da documentalista. In cambio lei sorveglia con un'attenzione gelosa i suoi manoscritti che legge, ascolta e ricopia per lui, cosa che rappresenta per lei una maniera di appropriarseli. Le sue testimonianze importanti permettono di comprovare o rettificare, su numerosi punti, la cronologia della redazione, ma anche di seguire la genesi editoriale e la ricezione delle opere, soprattutto di quelle teatrali. 